\title{
Interval Type-2 Fuzzy Logic Washing Machine
}

\author{
D. Nagarajan ${ }^{1}$, M. Lathamaheswari ${ }^{1}$, J. Kavikumar ${ }^{2}$, and E. Deenadayalan ${ }^{3}$ \\ ${ }^{1}$ Department of Mathematics, Hindustan Institute of Technology \& Science, Chennai, India \\ ${ }^{2}$ Department of Mathematics and Statistics, Faculty of Applied Science and Technology, Universiti Tun \\ Hussein Onn, Malaysia \\ ${ }^{3}$ Department of Electrical and Computer Engineering, Dire Dawa University, Ethiopia
}

\section{]jfis}

\begin{abstract}
By using fuzzy logic controller, one can control uncertain system where uncertainty exists on the input parameters. Generally, the delay in the system may cause time shift which disturbs the stability of the system. Therefore, the time delay systems with uncertainty can be represented by type-2 fuzzy logic controller. In this paper, the reference values are considered as intervals and automatic selection of detergent intake needed for good washing is proposed by using interval type- 2 fuzzy controller for the washing machine. Also transportation delay has been analyzed with the support of four different defuzzification methods and it is observed that $\mathrm{Wu}-\mathrm{Mendal}$ uncertainty bound method gives better result to keep the system stabilized than other methods.
\end{abstract}

Keywords: Control systems, Type-2 fuzzy logic controller, Transportation delay, Saturation, Detergent intake, Washing machine

\section{Introduction}

In the control systems, there are two major types such as proportional-integrated-derivative (PID) and fuzzy logic control systems (FLCSs) which are widely applied in the fields of engineering activities etc. Though PID controllers are universally used in the mechanism of control operations, it may exhibits a poor performance for nonlinear systems due to its insufficient knowledge of the parameters.

FLCS is an efficient model with several attributing qualities such as the reduced oscillations, better stability, small overshoot and faster settling time. To deal with parameter uncertainties, a mathematical tool was introduced by Zadeh in 1965 and suggested the soft computing. Linguistic constraints such as "many", "low", "medium", "often" and "few" can be represented by a mechanism provided by fuzzy theory. The approximate reasoning can be dealt with by applying the fuzzy logic systems (FLSs) and it is observed that the response of the system is better than PID with less control complexity.

In FLCS, a linguistic control strategy is converted into an automatic mode based on expert knowledge. The fuzzy logic (FL) provides control strategies for the low order dynamics with weak non linearities [1]. A type of differential equation in which the derivative of the uncertain function at a certain time is given in terms of the values of the function at preceding times is called a delay differential equation (DDE) and is also called time-delay systems. The delay will occur due to the usage of certain physical properties such 
as transmission of a signal and measurements of system variable.

The fuzzy rules are generally used in FLS and are often applied to capture the uncertainty lies in human reasoning [2]. The conventional control system needs an explicit model of the physical phenomenon whereas FL deals with problem based on the knowledge of the human operators [3]-5]. The theory of fuzzy provides a sufficient way for handling the uncertain criteria. In washing machine (WM), the stability analysis is an important task with respect to uncertain parameters. It is established on the spinning operation under peculiar condition for stress and it is the main source of the WM [6-8]. There is an interval type-2 fuzzy logic toolbox is available to get a desired output. Problem formation is based on if-then rule in terms of linguistic variable. Many defuzzification methods are being applied to maintain the stability of the system and also for getting a crisp output [8-13].

The rest of the paper is organized as follows: In Section 2, review of literature has been collected related to the present work. In Section 3, preliminaries of the proposed concept are given. In Section 4, the proposed concept and its process are explained in detail. In Section 5, comparative analysis is done with the existing methods. In Section 6, conclusion of the work and future direction is given

\section{Related Work}

[14] introduced interval type-2 fuzzy logic toolbox. The authors in [15], investigating about the first order linear fuzzy time delays dynamical system with the usage of alpha level sets. [16] proposed a method to solve an uncertain DDE and proved the existence and uniqueness of theorem (EUTs) by using Lipschitz condition and condition of linear gain by Banach fixed-point theorem. [1] used Liu process and proved a local EUT for fuzzy delay differential equation (FDDE) and developed a continuous dependence of the result corresponding to an initial data. [17] compared traditional PID controller and fuzzy logic controller for controlling liquid flow. [18] attained the solution of the EU to unbiased uncertain DDEs at state space $C_{q}([-q, 0] ; \mathbb{R})$.

[19] introduced a new analytical method for solving fuzzy linear DEs of order $n$. [20] used adapting fuzzy parameter and proposed a fuzzy delay predator-prey system. [2] investigated the fuzzy flexible control algorithm for multivariable system with uncertainty. [21] proposed the EUTs for fuzzy time delay dynamical system. [22] decisive optimal parameters to develop an ephemeral feedback of the PID control system. [23] enforced linear matrix inequality (LMI) in stabilizing the fractional order chaotic systems with uncertain parameters. [24] designed a washing machine using fuzzy logic. [25] proposed Frank aggregation operators for interval type-2 triangular fuzzy numbers and applied them in decision making problem.

[26] proposed an algorithm of the approximate mechanism for solving FDDE via Adomian decomposition method. [27] inclined an established statement to solve initial value problems for linear fuzzy interval Des using a generic representation under the consolidation of differences. [28] imported and examined two set of system of fuzzy differential formations.

[29] examined existence and uniqueness theorems and the properties of assured nonlinear FVIEs under SGD. [30] proposed emulating kernel Hilbert space method to get an explicit and analytical solutions of FVIE.

[31] commenced a new approach to solve FDE established on the reproducing kernel theory, inquiring, closed solutions for the equations of order two, boundary value problems for two points based on strongly generalized differentiability (SGD). [3] inquired some ideal results on fuzzy fractional differential equations. [4] used AFNN to design system for an execution of the decision for Washing Machine. [5] proposed existence and uniqueness of fuzzy solutions for the non-linear second order fuzzy Volterra Integrodifferential equations. [6] prompted a control system established on improved FL for WMs and proved that its taking less time for washing. [7] proposed a system for automatic filling of water and detergent for WM. [8] suggested a WM along with decay of power and water. [9] designed and established a smart WM based on FL. [10] applied fuzzy Sumuder transform (FST) method to approximate the solutions of FDEs. [11] developed a method to evaluate the enduring performance of WM. [12] applied fuzzy logic in the model of smart washing machine. [13] presented fuzzy logic control of washing machine.

\section{Preliminaries}

In this section some basic concepts related to the present is given.

\subsection{Fuzzy Set}

An extended version of a crisp set is called FS (fuzzy set). It allows a partial membership which takes values from 0 to 1 and is described by $\mu_{M}(x): X \rightarrow[0,1]$, where $M$ is a set, $\mu_{M}$ is the membership function (MF) and $X$ is the universal set. 


\subsection{Delay Differential Equation}

A DDE problem has the form

$$
\begin{aligned}
\dot{r}(t) & =f\left(t, r\left(t-\tau_{1}\right), \ldots, r\left(t-\tau_{n}\right)\right), t \geq t_{0}, \\
r(t) & =\gamma(t), t \leq t_{0},
\end{aligned}
$$

where $r(t)$ is the physical quantity which changes over the time and depend also on the past. $\phi(t)$ is the history function (HF). There may be one or more solutions depending on the regularity of HF.

\subsection{Types of Delays}

Generally there are four types of delays defined.

(i) Constant delay ( $\tau=$ constant $)$

(ii) Time reliant delay $(\tau=\tau(t))$

(iii) State dependent delay $(\tau=\tau(t, r(t)))$

(iv) Neutral delay $(\tau=\tau(t, r(t), \dot{r}(t)))$

\subsection{Fuzzy Delay Differential Equation}

Let $\wp$ be the Liu process and for some given functions $A, B$ : $[0$, $\infty) \times \wp_{q} \rightarrow \mathbb{R}$ the FDDE is

$$
d X(t)=A\left(t, X_{t}\right) d t+B\left(t, X_{t}\right) d \wp(t), t \geq \tau,
$$

where $X(t)=\gamma(t-\tau), \tau-q \leq t \leq \tau$.

If the given functions are continuous then $X:[\tau-q, b) \times$ $\Omega \rightarrow \mathbb{R}$ is a solution of FDDE if and only if

$$
X(t)=\left\{\begin{array}{c}
\phi(t-\tau) \\
\tau-q \leq t \leq \tau \\
\gamma(0)+\int_{\tau}^{t} A\left(s, X_{s}\right) d s+\int_{\tau}^{t} B\left(s, X_{s}\right) d \wp(s), \\
\tau \leq t \leq b .
\end{array}\right.
$$

The solution is an unsure process $X_{t}$ that contents the above equation identically in time $t$. Since many of the real world problems contain uncertainty in nature, the dynamical systems are designed by FDDE. If the basic design of the miniature depends upon the subjective preferences then fuzzy differential equation is the one way of incorporating the fuzziness exists in the system.

\subsection{Fuzzy Delayed Control System}

For each $s=1,2, \ldots, r$, a fuzzy delayed control system (FDCS) is defined by the plant rule

$$
\begin{gathered}
s: \text { IF } \delta_{1}(t) \text { is } M_{1 s} \text { AND } \ldots \text { AND } \delta_{p}(t) \text { is } M_{h s} \text { THEN } \\
\dot{x}(t)=E_{s} x(t)+F_{s} x\left(t-\tau_{s}(t)\right)+G_{s} u(t),
\end{gathered}
$$

where $\delta_{1}(t), \ldots, \delta_{h}(t)$ are the proposition variables and every $M_{i s}(i=1,2, \ldots, h ; s=1,2, \ldots, r)$ is the FS with respect to $\delta_{i}(t)$ and $s$. Let $M_{i s}\left[\delta_{i}(t)\right]$ be the MF of $M_{i s}$ at the position $\delta_{i}(t)$ and is defined by

$$
\omega_{s}[\delta(t)]=\prod_{i=1}^{h} M_{i s}\left[\delta_{i}(t)\right], \quad s=1,2, \ldots, r
$$

The resulting delayed FCS is concluded as the weighted average of the local model for the given pair of $[x(t), u(t)]$

$$
\begin{aligned}
\dot{x}(t)= & \frac{\sum_{s=1}^{r} \omega_{s}[\delta(t)]\left[E_{s} x(t)+F_{s} x\left(t-\tau_{s}(t)\right)+G_{s} u(t)\right]}{\sum_{s=1}^{r} \omega_{s}[\delta(t)]} \\
= & \sum_{s=1}^{r} k_{s}[\delta(t)]\left[E_{s} x(t)+F_{s} x\left(t-\tau_{s}(t)\right)+G_{s} u(t)\right], \\
& t \geq 0,
\end{aligned}
$$

where

$$
\begin{aligned}
& k_{s}[\delta(t)]=\frac{\omega_{s}[\delta(t)]}{\sum_{i=1}^{r} \omega_{i}[\delta(t)]}, \\
& k_{s}[\delta(t)] \geq 0, \quad \sum_{s=1}^{r} k_{s}[\delta(t)]=1 .
\end{aligned}
$$

In the local system $\dot{x}(t)$,

$$
\begin{aligned}
& x(t)=\left[x_{1}(t), x_{2}(t), \ldots, x_{n}(t)\right]^{T}, \\
& E_{s}=\left(e_{i j}^{s}\right)_{n \times n}, \quad F_{s}=\left(f_{i j}^{s}\right)_{n \times n}, \quad G_{s}=\left(g_{i j}^{s}\right)_{n \times m} .
\end{aligned}
$$

Using MFs, the delayed local systems are evenly linked to design a universal nonlinear delayed FDCS. For every system of delayed FCS, there must exist a constant $\tau>0$ such that $0 \leq \tau_{s}(t) \leq \tau, s=1,2, \ldots, r$. Assume the initial value for every solution to be $x(t)=\gamma(t), t \in[-\tau, 0]$. The vector continuous function is $\delta(t)=\left[\delta_{1}(t), \delta_{2}(t), \ldots, \delta_{n}(t)\right]^{T}$ and

$$
\|\gamma\|=\sup _{-\tau \leq \theta \leq 0} \sqrt{\gamma_{1}^{2}(\theta)+\gamma_{2}^{2}(\theta)+\cdots+\gamma_{n}^{2}(\theta)} .
$$




\subsection{Construction of Fuzzy Logic}

There are three distinct types of structures in constructing FL namely fuzzy sets (FSs), fuzzy variables (FVs), and fuzzy rules (FRs). A FS is decisive by a MF of a FV which generates values between 0 and 1 . The relationship between the antecedent and consequent FVs will actuate FR and are generally described linguistically by ordinary etymological terms. A rule accomplice an action about linguistic variables to a consequence is called fuzzy If-Then rule.

\subsubsection{Fuzzy logic controller}

Fuzzy engine has the tendency of imitating human behavior. To establish practical control systems, it is found that Fuzzy logic is the most fruitful automation which empowers control engineers to progress control planning in a potential manner. A system which is convoluted, poorly defined, and nonlinear or time shifting can be handled by fuzzy logic control (FLC) and hence there is a necessity of having mathematical excellence of the control system to implement FLC which is comparably accessible.

A FLC has three different parts namely fuzzifier, rule base and defuzzifier. The performance of each case is described as follows. Fuzzifier converts the numeric value into FSs and hence the process is called fuzzification. An essential component of the FLC is the inference engine, which executes all logic administrations. The rule base dwells with MFs and control rules. Finally, the defuzzifier revamps the FS into a numeric value as one should get a numeric output and hence the procedure is called defuzzification.

\subsubsection{Reason of using fuzzy logic}

In controlling the system, even though neural network and genetic algorithms are performing well in real world applications, the fuzzy logic is the most applicable technique as it has the convenience of obtaining solution where human operators can perceive and apply their skill in constructing the controller with automatic operations.

\subsubsection{Type-1 FLC}

In Type-1 FLC, input variables are mapped into FSs (sets of MFs). The MF is the graphical portrayal of the degree of every input and ultimately completes an output response. The nature of the MFs is supposedly based on numerical character and the extension of MFs. FR will be followed in the form of If-Then statement. Here, the membership values of an input will be considered as carrying the aspect for regulating their effect on the fuzzy output as sets in the final result. Once the function that runs the system are fixed, proportioned and connected then the crisp output will be occurred after defuzzification.

\subsubsection{Type-2 FLC}

It is proposed by Mendel and Liang. In type-2 fuzzy logic (T2FL) there are two types of membership grades (MGs) namely primary and secondary which takes values from 0 to 1 . For every primary grade there is a secondary MG which defines the possibilities of primary MGs. Generally in the interval T2FL system (IT2FLS) both primary and secondary MGs will be the interval sets. Since T2FL system has an intensive computational part it is widely applied in control system for the smooth performance.

T2FLC system has two kinds of MFs namely lower and upper which are represented by T1FS. T2FS also meant for interval based settings. The interval between superior and inferior MFs called footprint of uncertainty (FOU) and which represents the uncertainty level of the problem.

\subsubsection{Fuzzy inference system}

To handle a system with uncertain inputs or uncertain system, ordinary mathematical mechanism like differential equation is not suitable as the human decision is always uncertain in nature. By disparity, the subjective conditions of human knowledge and reasoning technique can be designed by fuzzy inference system (FIS) without engaging explicitly with quantitative investigation called FIS or fuzzy modeling or fuzzy identification. The core part of FIS is If-Then rules. Which is also called fuzzy conditional statements expressed by the form IF $P$ THEN $Q$, where $P$ and $Q$ are the fuzzy set labels represented by MFs appropriately. FRs also engaged the sequence of If-Then statements.

For example, by considering the following statement, if pressure is high then volume is small. Where the pressure and volume in antecedent part are lingual variables, high and small in the consequent part are the linguistic values or labels defined by MFs.

By using Takagi-Sugeno fuzzy model (TSFM), another fuzzy If - Then rule for the resistant force on a moving substance can

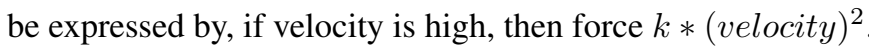
In this statement the antecedent part is again high which is fuzzy and the consequent part is a non-fuzzy equation of an input variable velocity. Both of the above cases have been 
applied in modeling as well as in controlling of the system extensively.

The complex nonlinear system can be represented by fuzzy sets and the fuzzy reasoning is using an impressive method called TSFM. In addition, it is an interesting and a smooth model since it allows an easy association of local linear system for designing the global nonlinear systems by using MFs. In the field of fuzzy control, some designing methods were introduced along with an expansion of fuzzy systems.

\subsubsection{Effects of delays in control system}

The existence of delays usually become the source of instability and deteriorating performance of the systems and therefore the stability of the system depends on the delay. Equipment of the system, transmission of the signal, size of the system variable, actuators, sensors and the field networks are reasons for getting delays in the control system.

Time delay: A system with time delay performs as a class of infinite dimensional system and used to depict the reproduction and transit development or population gesture. Nonlinear systems with time delay can be analyzed by the typical method called linearization and fuzzy logic theory for stabilizing the system. The designed controllers assure asymptotic stability, where the stability occurs while the component of the control system is operating but also when component failure exists as well. Usually time delay occurs due to the failing in transit and delay in collaborating the assessment sensor and actuator of the control. It is a serious problem and shall be dealt with appropriate measure otherwise it will affect the stability of the system. Since the outcome and effects are bound to happen determined by some time interval, so the delay in economic activity is natural. This kind of delay occurs often in biological system, network systems, rolling mill systems and metallurgical processing systems.

Neutral delay: The system with neutral delay also affects the stability and therefore it becomes a more complex issue as it affects the derivative of the delayed state. It can be handled by using linear matrix inequality (LMI), which is able to solve stability analysis and stabilization. Using LMI, the stability of the system can be analyzed for FCSs with bounded fuzzy delays. Here the results are independent of the length of the time delay. Neutral DDE can be applied when the dynamical system established on present and past states and involve derivatives with delay.

State delay: The delay in state and/or input of the variable also induces complex behavior of the system and obviously leads to stability problems.

Sensor delay: Sensor delay is called as a system along with outpouring controller and unanimity feedback although employing an output sensor with $\tau$ seconds late in releasing an output.

Actuator delay: If the delay arises while transfer the output of the controller then the delay is called actuator delay.

\subsubsection{Fuzzy logic in washing machine}

In our daily life, WM is one of the important appliances for household chores. The amount of washing time consuming is always based on the factors such as the type, the grade of dirt and also the number of cloths. This will be regulated by the sensors. But there is no standard mathematical concurrence between these parameters. In the early time, we tend to set an inexact time put to allocate for the running of WM, so we had the experience of error based on it. The controller regulates the outside input system as shown by sensors. This design can be recorded by fuzzy logic to help the system in making decisions similar to human behaviors by using interval type- 2 fuzzy logic controller (IT2FLC).

\subsubsection{Saturation time}

The saturation time of stain in the water and for vice versa is calculated incoherently with unquantifiable water level through visible sensor. The inferred visual sensor is measured on the basis of fuzzy rule. The time of saturation can be effectively measured by using IT2FC.

\section{Automatic Selection of Detergent Quantity Using Interval Type-2 Fuzzy Logic Controller}

The problem of automatic selection of the quantity of detergent according to the amount of cloths and their level of dirtiness is done by using IT2FLC.

\subsection{Problem Formation}

While using the WM, it calculatedly use the amount of washing powder or detergent manually based on the intelligence about the parameters such as the amount of cloths desired to wash and the type of the material as well as degree of dirt of the cloths.

Depending on the decision of the user for the selection of the detergent quantity, as a result the uncertainty exists. Hence to automate this process, we address the problem by using or 
applying the IT2FLC. The reason of using this type of fuzzy logic controller is that, the controller provides the exact amount of detergent even though an exact model of the input/output is not available. Here the parameters are considered as interval data instead of crisp one.

\subsection{Details of the Problem}

The problem of this paper is made comprehensible by considering only two variables.

The two inputs are weight of the cloths and type of dirt.

Figure 3 shows the basic methodology of the problem. The IT2FC takes these two inputs in the form of interval data, measures the information and the output of the quantity of the detergent. At the same time, getting the sensorial inputs of the WM and their working process (electrical, optical or any other type) is not the concern of this paper.

Introduction of the inputs is given below: the weight of the cloths decided by the knowledge of the person who is using the washing machine. The more amounts of the cloths necessitates the more consumption of the detergent. The type of dirt is determined by the saturation time, the time to reach saturation. The point of saturation is a point at which there is no further detectable changes in the color of water. The type of dirt determines the quality of dirt such as the excess level of grease, the medium level of grease or no content of grease at all.

Greasy cloths take large amount of detergent according to clearness level of the water since greasy type needs more amount of detergent for an effective washing process than the dirt of other types. Hence, the straight forward sensor system can supply us the necessary input for the IT2FLC.

\subsection{Plan of the Set Applied}

The range of possible values for the input and output variables need to be determined before dealing with details of IT2FLC. It is the MF which connects the real world calculations with fuzzy rules.

Figure 3 exhibits the labels of input and output variables and their corresponding MFs. The values of the input variables are assigned (-1 to 100) over the domain of optical sensor. The decision of the IT2FLC is derived from the rules which are saved in the database. Generally, the rules are supposedly IfThen statements which are spontaneous and understandable (common English statements).

Here the rules used are derived from the knowledge of an individual by using his/her common sense in the form of if-then statement like If (linguistic term) and (another linguistic term) then (linguistic term). Here the linguistic term is the result of the natural language with inherently uncertainty exists.

The set of rules are:

1) If the weight of cloths is more and type of dirt is greasy then the detergent intake is very high $(\mathrm{VH})$

2) If the weight of cloths is medium and type of dirt is greasy then the detergent intake is high $(\mathrm{H})$

3) If the weight of cloths is low and type of dirt is greasy then the detergent intake is low $(\mathrm{H})$

4) If the weight of cloths is high and type of dirt is medium then the detergent intake is high $(\mathrm{H})$

5) If the weight of cloths is medium and type of dirt is medium then the detergent intake is medium (M)

6) If the weight of cloths is low and type of dirt is medium then the detergent intake is low $(\mathrm{L})$

7) If the weight of cloths is high and type of dirt is not greasy then the detergent intake is medium (M)

8) If the weight of cloths is medium and type of dirt is not greasy then the detergent intake is low $(\mathrm{L})$

9) If the weight of cloths is low and type of dirt is not greasy then the detergent intake is very low (VL)

Here the level of greasiness is the reason for consuming the higher quantity of detergent. The case, not greasy which is mentioned in the above fuzzy rules has been considered as low and medium level of dirtiness of the cloths respectively for the calculation.

\subsection{Algorithm for Rule Calculation}

Step 1: Assume the range of the inputs as interval type-2 fuzzy numbers.

Step 2: Find the score values of the inputs.

Step 3: Assume the values lie in $[1.5,2]$ for high and maximum value 2 for very high, values lies in $[1,1.5]$ for medium and $[0,1]$ for low and the values lies in $[0,0.2]$ for very low. The resultant value is the level of detergent intake.

Step 4: End.

\subsection{Application of the Proposed Algorithm}

In Table 1, the weight and type of dirtiness of the cloths are considered as interval type- 2 triangular fuzzy numbers for the levels of low, medium and high. Detergent intake is calculated 
Table 1. Range of the input assumed for weight and dirt

\begin{tabular}{cc}
\hline Weight of cloths & Type of dirt \\
\hline L: $([0.2,0.3], 0.4,[0.5,0.6])$ & L: $([0.24,0.36], 0.47$, \\
& $[0.58,0.67])$ \\
M: $([0.4,0.5], 0.6,[0.7,0.8])$ & M: $([0.45,0.55], 0.66$, \\
& $[0.73,0.84])$ \\
H: $([0.6,0.7], 0.8,[0.9,0.95])$ & $\mathrm{H}:[0.67,0.79], 0.85$, \\
& $[0.92,0.97])$ \\
\hline
\end{tabular}

by the score function formula for triangular interval type- 2 fuzzy number (Table 2). Maximum value 2 denotes the very high intake and minimum value 0.1 denoted very low intake of detergent.

\section{Numerical computation:}

To find the output for the first rule:

Find the score value (SV) using score function (SF) of the triangular interval type-2 fuzzy number for each input and apply centroid method for getting a crisp output defined as follows [14].

$S(F)=\left(\frac{A_{l}{ }^{L}+A_{r}^{U}}{2}+1\right) \times\left(\frac{A_{l}^{L}+A_{l}^{U}+A_{r}{ }^{L}+A_{r}{ }^{U}+4}{8}\right.$

$S V([0.6,0.7], 0.8,[0.9,0.95])=1.4$,

$S V([0.67,0.79], 0.85,[0.92,0.97])=1.5$,

Detergent intake $=2$,

Similar computation for other rule set.

\subsection{Description of the System}

Two inputs dirt, weight and one output designed in fuzzy inference system. The dirt level and weight of the cloth are divided into three stages such as low, medium and high and are represented by triangular interval type-2 fuzzy numbers.

The fuzzy inference system was developed for interval type-2 fuzzy and named as IT2_FPID washing machine and 9 rules were framed. The Plant, Figure 1 is a process, fuzzy inference system is named as interval type-2 fuzzy PID, output response is $y$ and time delay denoted by block L. The time delay can be varied in the program using variable $\mathrm{L}$.

The sub system is shown in Figure 2 has fuzzy inference system and other blocks with variables which are represented in program scripts.

Figure 3 reveals Sugeno fuzzy inference system with membership functions where the editing of fuzzy rule is possible. Here two inputs give nine potential considerations.

The overall input membership and output memberships are

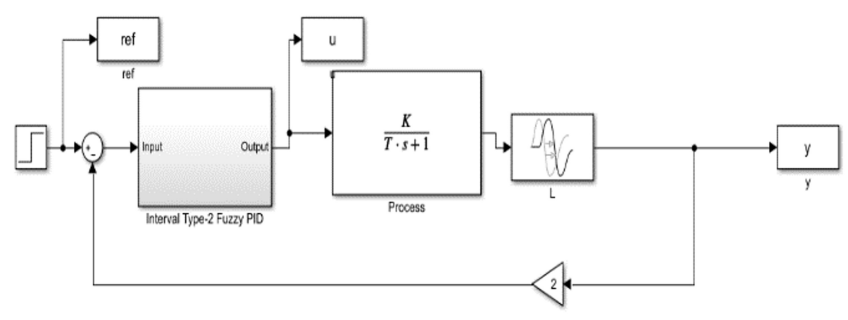

Figure 1. Block diagram of IT2F washing machine contol system.

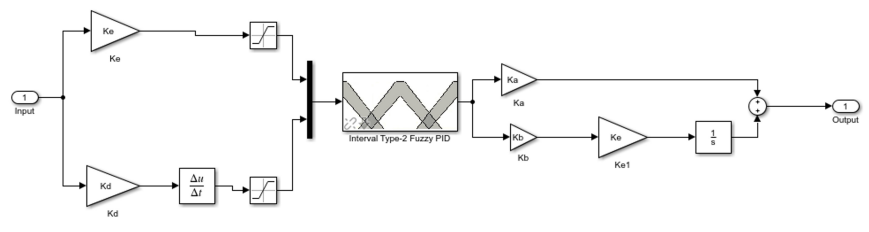

Figure 2. Block diagram of T2FC sub-system.

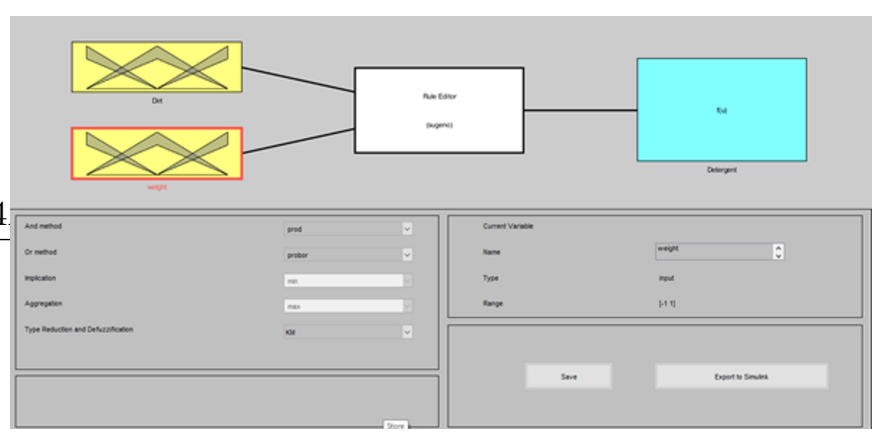

Figure 3. T2FC parameters.

given in Table 3.

Hence, the intake of the detergent varies according to the weight of the cloths and dirtiness of the cloths.

\subsection{Stabilize an Output with Reference}

To increase the stability of the output, there are four defuzzification methods used namely: Karnik-Mendel Algorithm (KM), Wu-Mendel uncertainty bound method (WM), Nie-Tan method (NT) and Begain-Melak-Mendel method (BMM) through TR method to analyze delay in transportation.

The stabilize the value of $K e=1, K d=0.5141, K a=$ $0.077, K b=17.336$, sampling time $=0.05, K=1, T=2.1$, and $L=0.01$.

Figure 4 shows that the different type of responses using KM, WM, NT, and BMM through TR method time with reference value. From the observation made, it is found that WM gives better performance for automatic detergent intake. 
Table 2. Interval type-2 fuzzy rule set

\begin{tabular}{ccccc}
\hline \multirow{2}{*}{ Rule No. } & \multicolumn{2}{c}{ Inputs } & Output \\
\cline { 2 - 4 } & Weight of cloths & Type of dirt & Detergent intake \\
\hline 1 & H: $([0.6,0.7], 0.8,[0.9,0.95])$ & H: $([0.67,0.79], 0.85,[0.92,0.97])$ & VH: 2 \\
2 & M: $([0.4,0.5], 0.6,[0.7,0.8])$ & H: $([0.67,0.79], 0.85,[0.92,0.97])$ & H: 1.5 \\
3 & L: $([0.2,0.3], 0.4,[0.5,0.6])$ & H: $([0.67,0.79], 0.85,[0.92,0.97])$ & H: 0.44 \\
4 & H: $([0.6,0.7], 0.8,[0.9,0.95])$ & M: $([0.45,0.55], 0.66,[0.73,0.84])$ & H: 1.51 \\
\hline 5 & M: $([0.4,0.5], 0.6,[0.7,0.8])$ & M: $([0.45,0.55], 0.66,[0.73,0.84])$ & M: 1.03 \\
6 & L: $([0.2,0.3], 0.4,[0.5,0.6])$ & M: $([0.45,0.55], 0.66,[0.73,0.84])$ & L: 0.31 \\
7 & H: $([0.6,0.7], 0.8,[0.9,0.95])$ & M(not greasy): $([0.45,0.55], 0.66,[0.73,0.84])$ & M: 1.51 \\
\hline 8 & M: $([0.4,0.5], 0.6,[0.7,0.8])$ & L(not greasy): $([0.24,0.36], 0.47,[0.58,0.67])$ & L: 0.65 \\
9 & L: $([0.2,0.3], 0.4,[0.5,0.6])$ & M(not greasy): $([0.24,0.36], 0.47,[0.58,0.67])$ & L: 0.20 \\
\hline
\end{tabular}

Table 3. Rule base of IT2FLC

\begin{tabular}{ccc}
\hline Weight & Dirt & Detergent intake \\
\hline H & H & VH \\
M & H & H \\
L & H & H \\
H & M & H \\
M & M & M \\
L & M & M \\
H & M (Not greasy) & M \\
M & L (Not greasy) & L \\
L & M (Not greasy) & VL \\
\hline
\end{tabular}

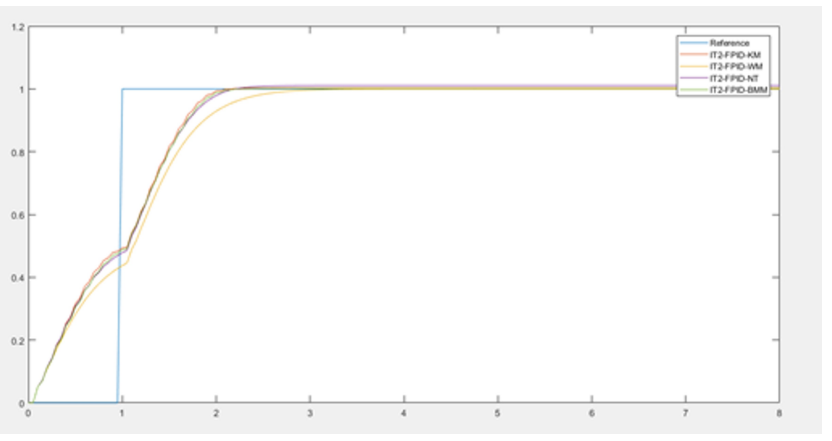

Figure 4. Settling point of the overall system response.

\subsection{Precision}

Figure 5 shows that the fuzzy rule based subsets are modified and reshaping the sub degree within the subset. Fuzzy knowledge based on fuzzy logic gives much better outcome. The output of the model shows that the detergent intake is varying according to the inputs.

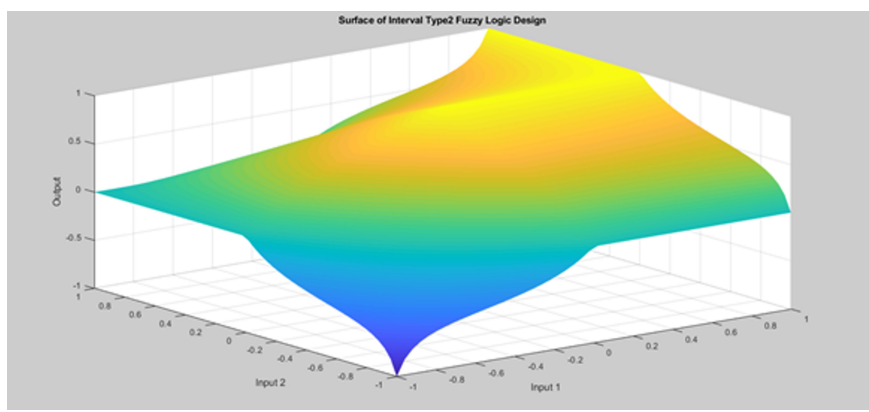

Figure 5. Surface of interval type-2 fuzzy logic.

\section{Comparative Analysis}

Here a comparative analysis is presented for the proposed work with the existing methods which show the novelty and effectiveness of the proposed method (Table 4).

\section{Conclusion}

The system which has uncertain parameters will have the problem of maintaining the system's stability. This issue can be solved by fuzzy logic. Interval T2FL handles more uncertainty to keep the system stabilized as it can deal imprecise parameters for interval data. It has an impressive capability to conclude the output and it can be characterized by aggressive growth and decomposition with respect to time for the system having uncertain criterion. In this work, interval type-2 fuzzy logic controller is used for automatic detergent intake according to weight and dirt of the cloths automatically. Four defuzzification methods were executed to analyze the transportation delay and among which Wu-Mendel uncertainty bound method (WM) gives better result for maintaining the system the system stabi- 
Table 4. Comparative analysis

\begin{tabular}{|c|c|}
\hline Existing methods & Proposed concept \\
\hline $\begin{array}{l}\text { In [8], smart washing } \\
\text { machine is designed and } \\
\text { developed using type- } 1 \\
\text { fuzzy logic controller. }\end{array}$ & $\begin{array}{l}\text { Which is capable of an } \\
\text { automatic inputs and desired } \\
\text { output using type-1 fuzzy } \\
\text { logic controller. }\end{array}$ \\
\hline $\begin{array}{l}\text { In }[9] \text {, type- } 1 \text { fuzzy logic } \\
\text { control of washing machine } \\
\text { is analyzed. }\end{array}$ & $\begin{array}{l}\text { Procedure has been used for } \\
\text { fixing washing time } \\
\text { according to the type of } \\
\text { cloths. }\end{array}$ \\
\hline $\begin{array}{l}\text { In [11], rule based fuzzy } \\
\text { logic has been developed. }\end{array}$ & $\begin{array}{l}\text { The parameters are crisp } \\
\text { numbers and the desired } \\
\text { output is obtained in terms } \\
\text { of percentage and the } \\
\text { standard units like } \\
\text { temperature. }\end{array}$ \\
\hline Proposed method & $\begin{array}{l}\text { Interval type- } 2 \text { fuzzy logic } \\
\text { controller has been used to } \\
\text { automate the detergent } \\
\text { intake and stability has been } \\
\text { analyzed using four } \\
\text { different defuzzification } \\
\text { methods and concluded the } \\
\text { better method. Also the } \\
\text { parameters have been } \\
\text { considered as interval type- } 2 \\
\text { fuzzy numbers and the } \\
\text { desired output is obtained } \\
\text { using the score of the inputs }\end{array}$ \\
\hline
\end{tabular}

lized.

\section{Conflict of Interest}

No potential conflict of interest relevant to this article was reported.

\section{References}

[1] V. Lupulescu and U. Abbas, "Fuzzy delay differential equations," Fuzzy Optimization and Decision Making, vol. 11, no. 1, pp. 99-111, 2012. https://doi.org/10.1007/ s10700-011-9112-7

[2] A. Boulkroune, M. M'Saad, and H. Chekireb, “Design of a fuzzy adaptive controller for MIMO nonlinear timedelay systems with unknown actuator nonlinearities and unknown control direction," Information Sciences, vol. 180, no. 24, 5041-5059, 2010. https://doi.org/10.1016/j. ins.2010.08.034
[3] O. Abu Arqub, A. S. Mohammed, S. Momani, and T. Hayat, "Numerical solutions of fuzzy differential equations using reproducing kernel Hilbert space method," Soft Computing, vol. 20, no. 8, pp. 3283-3302, 2016. https: //doi.org/10.1007/s00500-015-1707-4

[4] S. Habib and M. Akram, "Decision-making system for washing machine using AIFNN,"' Mathematical Sciences Letters, vol. 4, no. 3, pp. 303-311, 2015.

[5] S. Momani, O. Abu Arqub, S. Al-Mezel, and M. Kutbi, "Existence and uniqueness of fuzzy solutions for the nonlinear second-order fuzzy Volterra integrodifferential equations," Journal of Computational Analysis \& Applications, vol. 21, no. 2, pp. 213-227, 2016.

[6] A. Agarwal, A., Mishra, and M. Dixit, "Design of an improved fuzzy logic based control system for washing machines," International Journal of Computer Applications, vol. 151, no. 8, pp. 5-10, 2016.

[7] K. T. Oo and T. Z. Soe, "Operation system of washing machine with fuzzy logic control system and construction of detergent box," International Journal of Science, Engineering and Technology Research (IJSETR), vol. 5, no. 9, pp. 2883-2888, 2016.

[8] S. P. L. Roseline, "Unit consumption under fuzzy logic control of washing machine," Relevance of Research Methodology in Computer Science, 2016, Available http://www.isca.co.in/SOUV_PROC/conference/ISBN\% 20\%20978-93-84659-10-3.pdf\#page=17

[9] R. F. Masood, "Application of fuzzy logic in design of smart washing machine," 2017, Available https://arxiv.org/ abs/1701.01654

[10] R. P. Agarwal, D. Baleanu, J. J. Nieto, D. F. Torres, and Y. Zhou, "A survey on fuzzy fractional differential and optimal control nonlocal evolution equations," Journal of Computational and Applied Mathematics, vol. 339, pp. 3-29, 2018. https://doi.org/10.1016/j.cam.2017.09.039

[11] R. Stamminger, P. Tecchio, F. Ardente, F. Mathieux, and P. Niestrath, "Towards a durability test for washing-machines," Resources, Conservation and Recycling, vol. 131, pp. 206-215, 2018. https://doi.org/10.1016/ j.resconrec.2017.11.014

[12] duplicate with [9] 
[13] M. Agarwal, "Fuzzy logic control of washing machines," 2011, Available https://pdfs.semanticscholar.org/ f4fb/586d70e66c9e592d8abade5f796fb4a5f7c6.pdf

[14] J. R. Castro, O. Castillo, and L. G. Martinez, "Interval type-2 fuzzy logic toolbox," Engineering Letters, vol. 15, no 1, pp. 1-10, 2007.

[15] M. H. Farahi and S. Barati, "Fuzzy time-delay dynamical systems," The Journal of Mathematics and Computer Science, vol. 2, no. 1, pp. 44-53, 2011.

[16] X. Ge and Y. Zhu, "Existence and uniqueness theorem for uncertain delay differential equations," Journal of Computational Information Systems, vol. 8, no. 20, pp. 83418347, 2012.

[17] A. K. Gaurav and A. Kaur, "Comparison between conventional PID and fuzzy logic controller for liquid flow control: performance evaluation of fuzzy logic and PID controller by using MATLAB/Simulink," International Journal of Innovative Technology and Exploring Engineering, vol. 1, no. 1, pp. 84-88, 2012.

[18] H. Liu, and W. Fei, "Neutral delay differential equations," Information, vol. 15, no. 12, pp. 359-366, 2012.

[19] S. Lata and A. Kumar, "A new analytical method for solving fuzzy differential equations," Iranian Journal of Fuzzy Systems, vol. 10, no. 4, pp. 19-39, 2013.

[20] N. Maan, K. Barzinji, and N. A. Aris, "Fuzzy delay differential equation in predator-prey interaction: analysis on stability of steady state," in Proceedings of the World Congress on Engineering, London, UK, 2013, pp. 3-5.

[21] N. A. I. Zulkefli and N. Maan, N. (2014). The existence and uniqueness theorems of fuzzy delay differential equations. Malaysian Journal of Fundamental and Applied Sciences, vol. 10, no. 3, pp. 139-143, 2014. https://doi.org/10.11113/mjfas.v10n3.280

[22] A. M. Shayma'a, "Optimization of Pid controller parameters based on genetic algorithm for non-linear electromechanical actuator," International Journal of Computer Applications, vol. 94, no. 3, pp. 11-20, 2014.

[23] B. Wang, J. Xue, and D. Chen, "Takagi-Sugeno fuzzy control for a wide class of fractional-order chaotic systems with uncertain parameters via linear matrix inequality," Journal of Vibration and Control, vol. 22, no. 10, pp. 2356-2369, 2016. https://doi.org/10.1177\% 2F1077546314546682

[24] M. Demetgul, O. Ulkir, and T. Waqar, "Washing machine using fuzzy logic," Automation, Control and Intelligent Systems, vol. 2, no. 3, pp. 27-32, 2014. http://doi.org/10. 11648/j.acis.20140203.11

[25] J. Qin and X. Liu, "Frank aggregation operators for triangular interval type-2 fuzzy set and its application in multiple attribute group decision making," Journal of Applied Mathematics, vol. 2014, article no. 923213, 2014. http://dx.doi.org/10.1155/2014/923213

[26] S. Narayanamoorthy and T. L. Yookesh, "Approximate method for solving the linear fuzzy delay differential equations," Discrete Dynamics in Nature and Society, vol. 2015, article no. 273830, 2015. http://dx.doi.org/10.1155/ $2015 / 273830$

[27] Y. Chalco-Cano, A. Khastan, and R. Rodriguez-Lopez, "Normalized expression for solutions to linear fuzzy differential equations under combination of differences," in Proceedings of 2015 Conference of the International Fuzzy Systems Association and the European Society for Fuzzy Logic and Technology (IFSA-EUSFLAT-15), Gijon, Spain, 2015. https://dx.doi.org/10.2991/ifsa-eusflat-15.2015.195

[28] C. Min, Z. B. Liu, L. H. Zhang, and N. J. Huang, "On a system of fuzzy differential inclusions," Filomat, vol. 29, no. 6, pp. 1231-1244, 2015. http://doi.org/10.2298/ FIL1506231M

[29] O. Abu Arqub, S. Momani, S. Al-Mezel, and M. Kutbi, "Existence, uniqueness, and characterization theorems for nonlinear fuzzy integrodifferential equations of Volterra type," Mathematical Problems in Engineering, vol. 2015, article no. 835891, 2015. http://dx.doi.org/10.1155/2015/ 835891

[30] O. Abu Arqub, "Adaptation of reproducing kernel algorithm for solving fuzzy Fredholm-Volterra integrodifferential equations," Neural Computing and Applications, vo. 28, no. 7, pp. 1591-1610, 2017. https://doi.org/10.1007/ s00521-015-2110-x

[31] O. Abu Arqub, M. Al-Smadi, S. Momani, and T. Hayat, "Application of reproducing kernel algorithm for solving second-order, two-point fuzzy boundary value prob- 
lems," Soft Computing, vol. 21, no. 23, pp. 7191-7206, 2017. https://doi.org/10.1007/s00500-016-2262-3

[32] T. K. Sai and K. A. \& Reddy, "Fuzzy applications in a power station," International Journal on Soft Computing, vol. 6, no. 2, pp. 1-16, 2015.

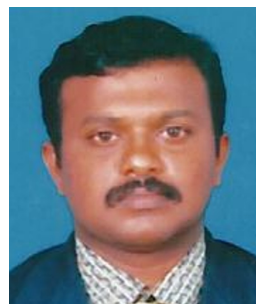

D. Nagarajan is a Professor in the Department of Mathematics, Hindustan Institute of Technology \& Science, Padur, Chennai. He has done his Ph.D. from Manonmaniyam Sundaranar University. His research interests are stochastic process, hidden Markov models, image processing and fuzzy mathematics.

E-mail: dnrmsu2002@yahoo.com

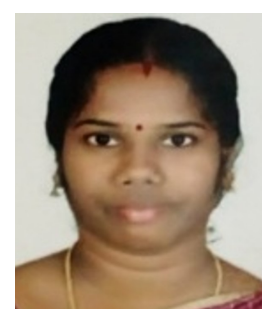

M. Lathamaheswari is Assistant Professor in the Department of Mathematics, Hindustan Institute of Technology and Science, Chennai. She has done her M.Phil. from Bharathidasan University, Tiruchirappalli. She is doing her research in fuzzy optimization under the guidance of Prof. Dr. D. Nagarajan in Hindustan Institute of Technology and Science, Chennai. E-mail: lathamax@gmail.com

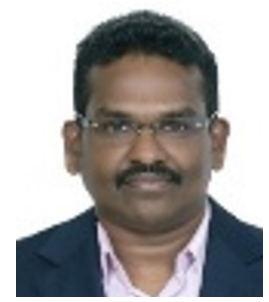

Kavikumar Jacob earned his M.Sc. (Mathematics) in 1999 and Ph.D. degree in Pure Mathematics from Annamalai University, India, in 2005. Since 2006 he has been at Department of Mathematics and Statistics, Faculty of Applied Sciences and Technology, Universiti Tun Hussein Onn Malaysia, Malaysia. His research interests include automata theory, fuzzy mathematics, and numerical analysis.

E-mail: kavi@uthm.edu.my

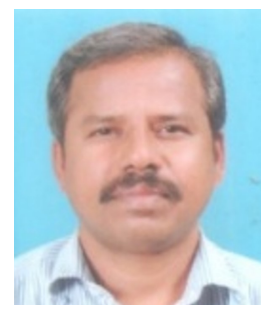

E. Deenadayalan is Lecturer in department of electrical and computer engineering, Dire Dawa University, Ethiopia. His research interests are instrumentation and control systems.

E-mail: deenaieee@yahoo.com 